\title{
Dark state experiments with ultracold, deeply-bound triplet molecules
}

\author{
Florian Lang, ${ }^{1}$ Christoph Strauss, ${ }^{1}$ Klaus Winkler, ${ }^{1}$ Tetsu Takekoshi, ${ }^{1}$ \\ Rudolf Grimm, ${ }^{1,2}$ Johannes Hecker Denschlag ${ }^{1 *}$ \\ ${ }^{1}$ Institut für Experimentalphysik und Zentrum für Quantenphysik, \\ Universität Innsbruck, A-6020 Innsbruck, Austria \\ ${ }^{2}$ Institut für Quantenoptik und Quanteninformation \\ der Österreichischen Akademie der Wissenschaften, A-6020 Innsbruck, Austria \\ *E-mail: johannes.denschlag @ uibk.ac.at
}

\begin{abstract}
We examine dark quantum superposition states of weakly bound $\mathrm{Rb}_{2}$ Feshbach molecules and tightly bound triplet $\mathrm{Rb}_{2}$ molecules in the rovibrational ground state, created by subjecting a pure sample of Feshbach molecules in an optical lattice to a bichromatic Raman laser field. We analyze both experimentally and theoretically the creation and dynamics of these dark states. Coherent wavepacket oscillations of deeply bound molecules in lattice sites, as previously observed in Ref. [1], are suppressed due to laser-induced phase locking of molecular levels. This can be understood as the appearance of a novel multilevel dark state. In addition, the experimental methods developed help to determine important properties of our coupled atom / laser system.
\end{abstract}

\section{Introduction}

Very recently, several groups have produced dense, ultracold ensembles of molecules that are deeply bound [1, 2, 3, 4, 5] and in a ro-vibrational ground state [1, 2, 4, 5]. This was achieved by binary association of alkali atoms in ultracold ensembles via two different pathways: (1) photoassociation [6, 7] and (2) magneto-association at Feshbach reso- 
nances [7, 8] combined with stimulated Raman adiabatic passage (STIRAP) [9], a special coherent optical transfer method. In contrast to photoassociation, magneto-association only produces weakly-bound Feshbach molecules [7, 8]. STIRAP can then be used to transfer these weakly-bound molecules to the rovibrational ground state. This method is coherent, efficient, fast, reversible, and highly selective. STIRAP is based on a counterintuitive light pulse sequence giving rise to a dynamically changing dark superposition state (Fig. 11 h)

$$
|D S\rangle=\left(\Omega_{2}|f\rangle-\Omega_{1}|g\rangle\right) / \sqrt{\Omega_{1}^{2}+\Omega_{2}^{2}} .
$$

In this paper, we deliberately replace the efficient but complex STIRAP transfer of Ref. [1] with a simple square laser pulse scheme. This reveals interesting fundamental processes and dynamics in the coupled atom / laser system, that would otherwise be hidden. In addition, this procedure allows us to determine important properties and parameters of our system and to check for consistency with our theoretical model. We study the creation and lifetime of dark superposition states that contain a sizeable fraction of deeply bound molecules. These molecules are held in a 3D optical lattice. Because the lattice potential is much shallower for the deeply bound molecules than for the Feshbach molecules, and because the transfer is fast, the deeply bound molecules coherently populate several Bloch bands. In contrast to Ref. [1], where similar circumstances lead to coherent oscillations in the lattice, oscillations are suppressed in the experiment described here due to phase locking of all quantum levels involved. A novel dark state appears which is a superposition of up to 8 quantum levels. We investigate the limiting conditions under which oscillations set in. 


\section{Experimental setup and initial preparation of molecules}

We carry out our dark state experiments with a $50 \mu \mathrm{m}$-size pure ensemble of $3 \times 10^{4}$ weakly bound $\mathrm{Rb}_{2}$ Feshbach molecules. The molecules are trapped in the lowest Bloch band of a cubic 3D optical lattice with no more than a single molecule per lattice site [10] and an effective lattice filling factor of about 0.3. The lattice depth for the Feshbach molecules is $60 E_{r}$, where $E_{r}=\pi^{2} \hbar^{2} / 2 m a^{2}$ is the recoil energy, with $m$ the mass of the molecules and $a=415.22 \mathrm{~nm}$ the lattice period. Such deep lattices suppress tunneling between different sites. A pure ensemble of Feshbach molecules has been produced as follows. We prepare a cold cloud of $6 \times 10^{5}{ }^{87} \mathrm{Rb}$ atoms that are either Bose condensed or nearly 1 Bose condensed in a Ioffe-type magnetic trap with trap frequencies $\omega_{x, y, z}=$ $2 \pi \times(7,19,20) \mathrm{Hz}$. Within $100 \mathrm{~ms}$ we adiabatically load the atoms into the 3D optical lattice. After turning off the magnetic trap, we flip the spins of our atoms from their initial state $\left|F=1, m_{F}=-1\right\rangle$ to $\left|F=1, m_{F}=+1\right\rangle$ by suddenly reversing the bias magnetic field of a few G. This spin state features a $210 \mathrm{mG}$-wide Feshbach resonance at $1007.40 \mathrm{G}$ [11]. By adiabatically ramping over this resonance, we efficiently convert atoms at multiply occupied lattice sites into $\mathrm{Rb}_{2}$ Feshbach molecules. After conversion, inelastic collisions occur at lattice sites that contain more particles than a single Feshbach molecule, leading to vibrational relaxation of these molecules, release of binding energy into kinetic energy and removal of all particles from these sites. A subsequent combined microwave and optical purification pulse removes all remaining chemically unbound atoms, creating a pure sample of $3 \times 10^{4}$ Feshbach molecules. Afterwards, the magnetic field is set to 1005.8 G, where the Feshbach molecules are in a quantum state $|f\rangle$ which correlates with $\left|F=2, m_{F}=2, f_{1}=2, f_{2}=2, v=36, l=0\right\rangle$ at 0 G. Here, $F$ and $f_{1,2}$ are the total angular momentum quantum numbers for the molecule and its atomic constituents, respectively,

\footnotetext{
${ }^{1}$ It turns out that this increases the number of Feshbach molecules.
} 

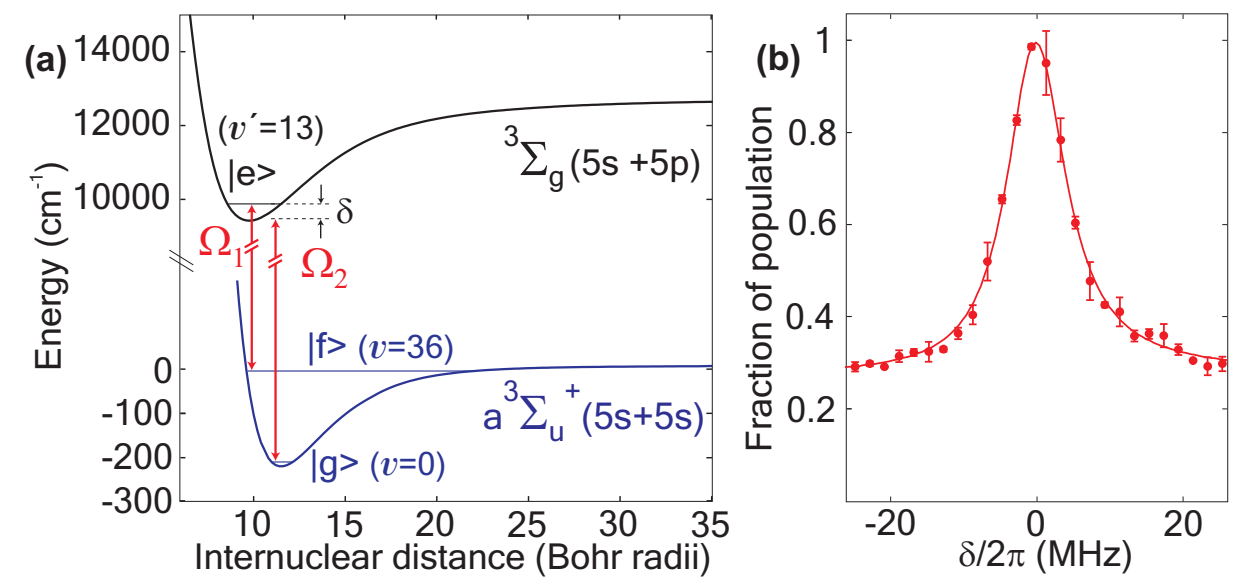

Figure 1: (a) $\Lambda$-type three-level scheme for dark states. The lasers 1 and 2 couple the molecular levels $|f\rangle,|g\rangle$ to the excited level $|e\rangle$ with Rabi frequencies $\Omega_{1,2}$, respectively. (b) Typical dark resonance. Shown is the fraction of Feshbach molecules $|f\rangle$ remaining after exposing them to both lasers in a $3 \mu$ s square pulse for varying two-photon detuning $\delta$. The data is identical to that shown in Ref. [1].

and $m_{F}$ is the total magnetic quantum number; $v$ is the vibrational quantum number for the triplet ground state potential $\left(a^{3} \Sigma_{u}^{+}\right)$and $l$ is the quantum number for rotation.

The bichromatic Raman laser field for the creation of the molecular dark states is based on two lasers (1 and 2) which connect the Feshbach molecule level $|f\rangle$, via an excited level $|e\rangle$, to the absolute lowest level in the triplet potential $|g\rangle$ (Fig.11 a). Laser 1 is a Ti:Sapphire laser and laser 2 is a grating-stabilized diode laser. Both lasers are PoundDrever-Hall locked to a single cavity which itself is locked to an atomic ${ }^{87} \mathrm{Rb}$-line. From the lock error signals, we estimate frequency stabilities on a ms-timescale of $40 \mathrm{kHz}$ and $80 \mathrm{kHz}$ for lasers 1 and 2, respectively. Both laser beams have a waist of $130 \mu \mathrm{m}$ at the location of the molecular sample, propagate collinearly, and are polarized parallel to the direction of the magnetic bias field. Thus, the lasers can only induce $\pi$ transitions.

The ground state $|g\rangle$ has a binding energy of 7.0383(2) $\mathrm{THz} \times h$ and can be described by the quantum numbers $\left|F=2, m_{F}=2, S=1, I=3, v=0, l=0\right\rangle$ where $S$ and $I$ are 
the total electronic and nuclear spins of the molecule, respectively. At $1005.8 \mathrm{G}|g\rangle$ is separated by hundreds of MHz from any other bound level, so that there is no ambiguity as to which level is addressed. The level $|e\rangle$ is located in the vibrational $v=13$ manifold of the electronically excited ${ }^{3} \Sigma_{g}(5 \mathrm{~s}+5 \mathrm{p})$ potential and has $1_{\mathrm{g}}$ character. It has an excitation energy of $294.6264(2) \mathrm{THz} \times h$ with respect to $|f\rangle$, and a width $\Gamma=2 \pi \times 8 \mathrm{MHz}$. The Rabi frequencies $\Omega_{1,2}$ of the two lasers depend on their respective intensities $I_{1,2}$, i.e., $\Omega_{1}=$ $2 \pi \times 0.4 \mathrm{MHz} \sqrt{I_{1} /\left(\mathrm{Wcm}^{-2}\right)}$ and $\Omega_{2}=2 \pi \times 30 \mathrm{MHz} \sqrt{I_{2} /\left(\mathrm{Wcm}^{-2}\right)}$, and are typically chosen to be in the $\mathrm{MHz}$ regime.

\section{Dark state evolution within a square pulse}

Our square pulse projection experiments are carried out as follows. We expose the Feshbach molecules $|f\rangle$ in the lattice to square pulses of Raman lasers 1 and 2 of variable pulse duration. Laser 2 is switched on about $1 \mu$ s before laser 1 to avoid excitation from $|f\rangle$ to $|e\rangle$ due to jitter in the laser pulse timing. The Raman lasers are resonant $(\delta=0)$ and the Rabi frequency $\Omega_{2} \approx 2 \pi \times 7 \mathrm{MHz}$ while $\Omega_{1}$ is varied (Fig. 2). After the pulse, we measure the fraction of molecules remaining in state $|f\rangle$ by dissociating them into pairs of atoms at the Feshbach resonance, releasing them from the lattice and applying standard absorption imaging. It is important to note that we actually only count atoms in the lowest Bloch band of the lattice. The release from the optical lattice is done as described in [12], where after 13 ms of ballistic expansion we map out the Bloch bands in momentum space (see Appendix for details).

Figure 2 shows the remaining fraction of molecules in state $|f\rangle$ versus pulse duration. Within $1 \mu$ s we observe a rapid loss of molecules that depends on the ratio $\Omega_{2} / \Omega_{1}$. The remaining molecules are stable on a much longer timescale. This can be understood in terms of formation of a dark state $|D S\rangle$. We can write 


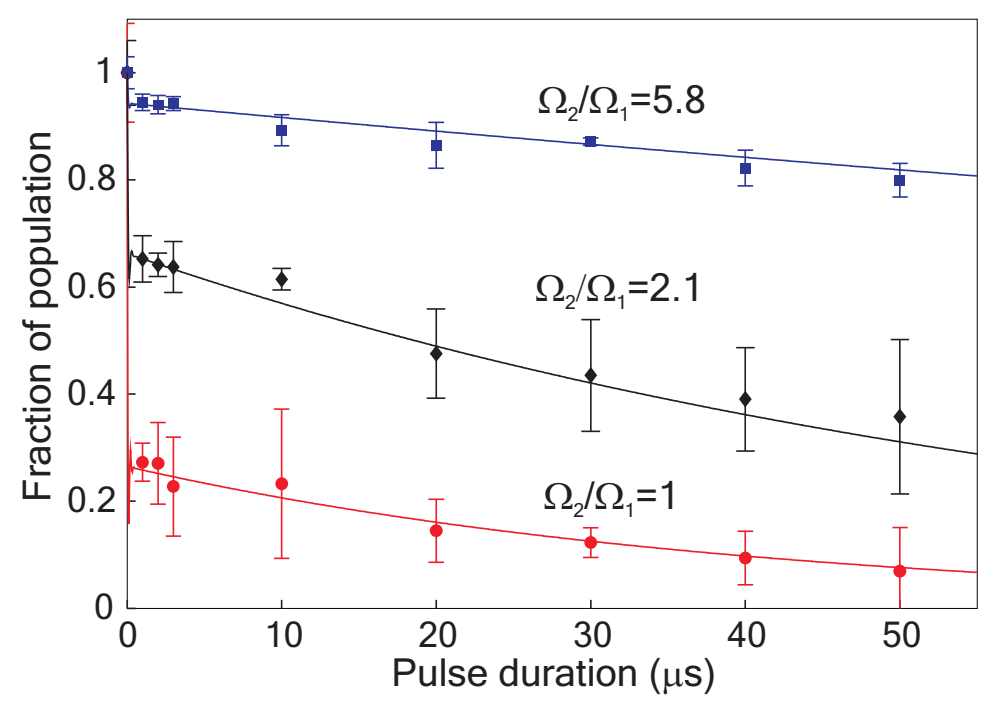

Figure 2: Dark state formation and lifetime. Shown is the fraction of Feshbach molecules remaining after subjecting them to a square pulse of Raman laser light of varying length for various Rabi frequency ratios $\Omega_{2} / \Omega_{1}\left(\Omega_{2} \approx 2 \pi \times 7 \mathrm{MHz}\right)$. After switching on the lasers, a certain fraction of molecules is lost within $1 \mu \mathrm{s}$ and a dark state has formed which has a much longer lifetime. The solid lines represent model calculations (Sec. (4) which can be used to determine the Rabi frequencies and short-term laser linewidths.

$$
|f\rangle=\left(\Omega_{2}|D S\rangle+\Omega_{1}|B S\rangle\right) / \sqrt{\Omega_{1}^{2}+\Omega_{2}^{2}}
$$

where

$$
|B S\rangle=\left(\Omega_{1}|f\rangle+\Omega_{2}|g\rangle\right) / \sqrt{\Omega_{1}^{2}+\Omega_{2}^{2}}
$$

is a bright state which quickly decays via resonant excitation to level $|e\rangle$. The dark state remains after the lasers are switched on and can be detected as a fraction $\Omega_{2}^{4} /\left(\Omega_{1}^{2}+\right.$ $\left.\Omega_{2}^{2}\right)^{2}$ of molecules projected back to $|f\rangle$ after switching off the laser.2.

Also, after the pulse a fraction $\Omega_{1}^{2} \Omega_{2}^{2} /\left(\Omega_{1}^{2}+\Omega_{2}^{2}\right)^{2}$ of the initial molecules are in state $|g\rangle$ with a maximum of $25 \%$ for $\Omega_{1}=\Omega_{2}$. Thus, a sizeable fraction of the molecules can

\footnotetext{
${ }^{2}$ This fact can be used to conveniently calibrate the Rabi frequency ratio $\Omega_{1} / \Omega_{2}$. We found good agreement with other calibration methods for the Rabi frequencies.
} 
be coherently transferred to the ground state. Remarkably, this transfer takes place in less than $1 \mu \mathrm{s}$ ! Such short transfer times cause Fourier broadening, resulting in considerably reduced laser stability requirements. In addition, due to the formation of a dark state, there is still a well-defined phase relation between the $|f\rangle$ and $|g\rangle$ molecules.

As can be seen from Fig. 2, the dark state slowly decays. Its lifetime is shortest for $\Omega_{1}=\Omega_{2}$, where we measure it to be $\approx 50 \mu \mathrm{s}$. The decay of the dark state is likely due to phase fluctuations of the Raman lasers. Phase fluctuations lead to an admixture of a bright state component to the otherwise dark state, which causes losses. In Sec. 4 we will show that these fluctuations can be expressed in terms of the short-term relative linewidth of the lasers, $\gamma$, which we find to be about $2 \pi \times 20 \mathrm{kHz}$. In principle, the decay of the dark state could be due to other effects, such as coupling to levels other than $|f\rangle,|e\rangle$, and $|g\rangle$. However, we have verified that this is not the case, because losses due to optical excitation are completely negligible on the $100 \mu$ s-timescale when we expose a pure ensemble of $|f\rangle$ $(|g\rangle)$ molecules to only laser 2 (1).

We also searched for laser power dependent shifts of the two-photon resonance. Using the Raman square pulse measurements, we scanned the relative detuning of the lasers for a fixed pulse duration and various laser powers. Within the accuracy of our measurements of $2 \pi \times 200 \mathrm{kHz}$, we could not detect any shifts of the resonance.

The behavior in Fig. 2] is described well by a closed three-level model (a $\Lambda$ system) and its dynamics can be simulated with a master equation which we describe in the following.

\section{Three-level model and master equation}

Neglecting lattice effects, we can describe the internal dynamics of the molecules as they are subjected to the Raman laser fields with a three-level model. We use a master equation [13, 14] which takes into account decoherence due to phase fluctuations of the Raman 


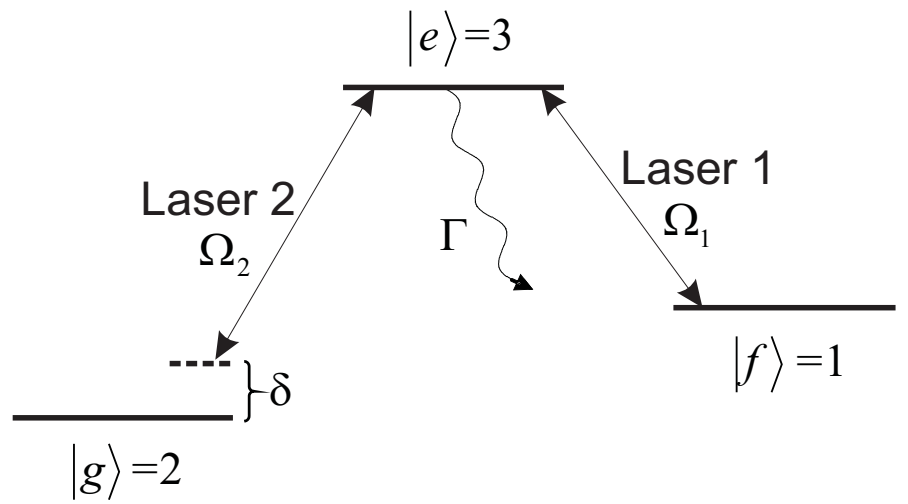

Figure 3: Level scheme for the master equation.

lasers. We consider the case where laser 1 is kept on resonance and laser 2 has a detuning $\delta$ (Fig. 3). Identifying the levels $|f\rangle,|g\rangle,|e\rangle$ with numbers 1, 2, 3, respectively, we can write the master equation as,

$$
\begin{aligned}
\frac{d \rho}{d t} & =-i \delta\left[\sigma^{22}, \rho\right]-\frac{i}{2} \sum_{k=1}^{2} \Omega_{k}\left[\sigma_{-}^{3 k}+\sigma_{+}^{3 k}, \rho\right] \\
& -\frac{1}{2} \Gamma\left(\sigma^{33} \cdot \sigma^{33} \cdot \rho+\rho \cdot \sigma^{33} \cdot \sigma^{33}\right) \\
& +\frac{1}{2} \gamma\left(2 \sigma^{22} \cdot \rho \cdot \sigma^{22}-\sigma^{22} \cdot \rho-\rho \cdot \sigma^{22}\right)
\end{aligned}
$$

where $\rho$ is the density matrix, $\Omega_{1,2}$ are the Rabi frequencies, $\Gamma$ is the spontaneous decay rate of the excited level $|e\rangle$, and $\gamma$ is the relative linewidth of the two Raman lasers. The matrices $\sigma_{-}^{r s}$ and $\sigma_{+}^{r s}$ are ladder operators and each is the transpose of the other. For example

$$
\sigma_{-}^{32}=\left(\begin{array}{ccc}
0 & 0 & 0 \\
0 & 0 & 0 \\
0 & 1 & 0
\end{array}\right)=\left(\sigma_{+}^{32}\right)^{\mathrm{T}}
$$

Setting the linewidth of the excited level $\Gamma=8 \mathrm{MHz}$, the detuning $\delta=0$ and Rabi frequencies $\Omega_{2}=2 \pi \times 7 \mathrm{MHz}$ and $\Omega_{1}$ to give the ratios in Fig. 2, we fit all the data with 


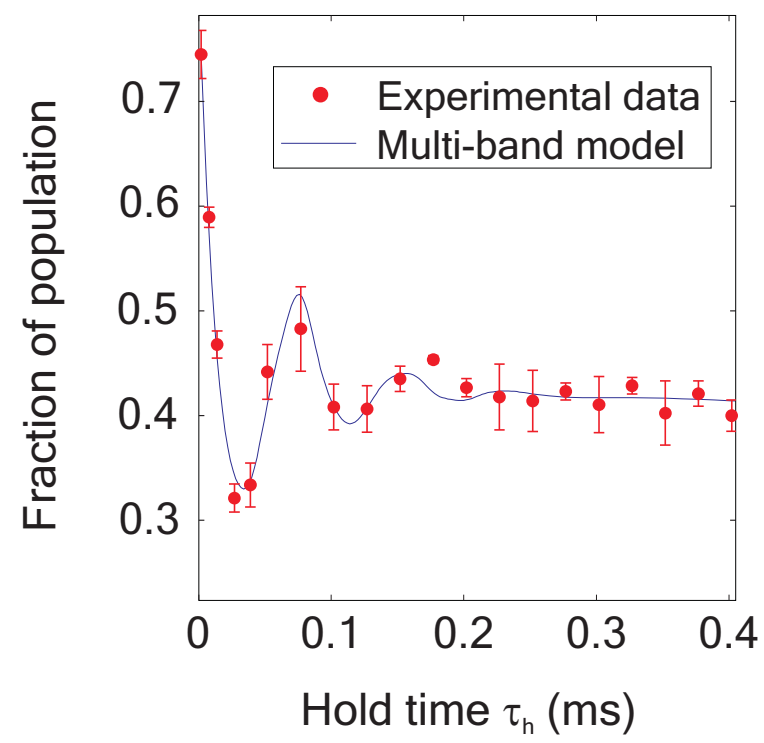

Figure 4: We plot the transfer efficiency for the round-trip STIRAP process as a function of the hold time $\tau_{h}$ between the two STIRAP pulses. With our procedure we only count molecules whose constituent atoms end up in the lowest Bloch band after transfer. The oscillations in the transfer efficiency are due to breathing oscillations of localized spatial wavepackets of molecules in the lattice sites. The solid line is from a multi-band model calculation (Sec. 6). This plot is taken from Ref. [1].

a single fit parameter $\gamma$. As a best fit, we obtain a relative linewidth of the two Raman lasers $\gamma=2 \pi \times 20 \mathrm{kHz}$, which is a reasonable value for our laser system.

\section{Coherent oscillations and their suppression}

In reference [1] coherent oscillations of molecular wavepackets of $|g\rangle$ molecules in the optical lattice were observed. We now investigate how these observations fit together with the experimental results of the square pulse projection experiments presented here. For clarity, the oscillation data from Ref. [1] are presented again in Fig. 4 and briefly discussed.

Using an STIRAP pulse sequence, Feshbach molecules are efficiently transferred to level $|g\rangle$. The Raman lasers are extinguished and the molecules are held for a time $\tau_{h}$, 


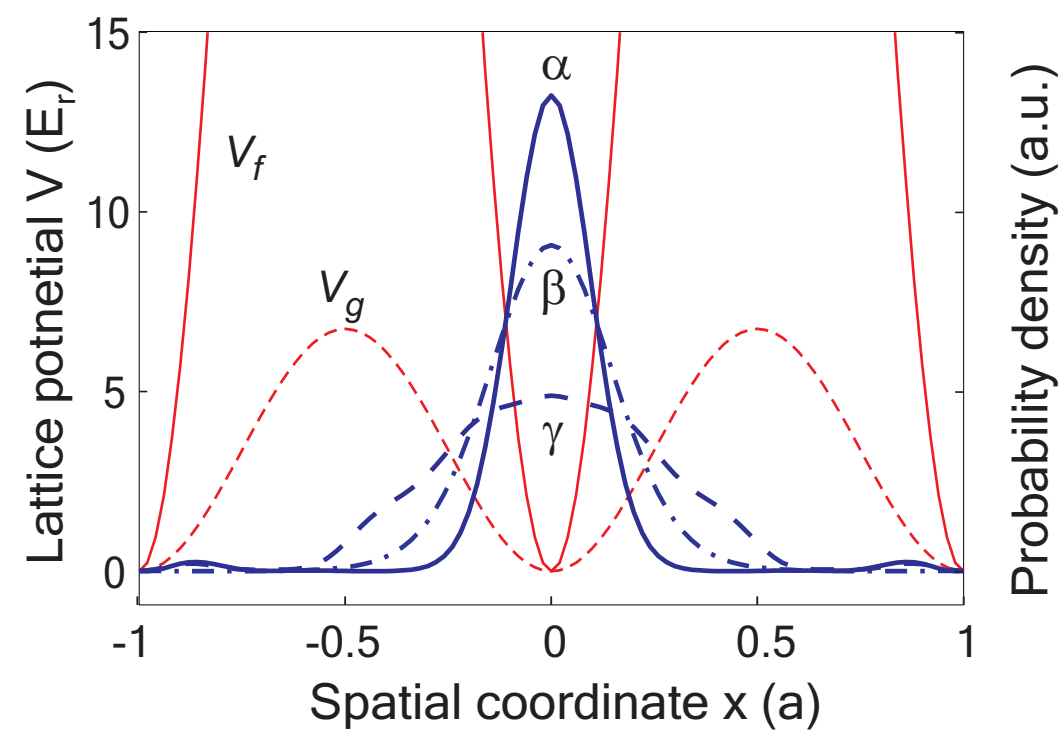

Figure 5: Wavepacket dynamics. Directly after the STIRAP transfer of a molecule from $|f\rangle$ to $|g\rangle$ the shape of its wavepacket (thick solid line $\alpha$ ) essentially corresponds to the vibrational ground state of the sinusoidal lattice potential for Feshbach molecules $V_{f}$ (thin solid line). In the much weaker potential felt by the ground state molecules $V_{g}$ (thin dashed line) the wavepacket starts to oscillate. After $1 / 8$ of the oscillation period $\tau_{o s c}=2 \pi / \omega_{t}$ its shape roughly corresponds to the vibrational ground state for $|g\rangle$ (thick dashed line $\beta$ ) and reaches its maximum extension after $\tau_{o s c} / 4$ (thick dash-dotted line $\gamma$ ).

after which they are transferred back to $|f\rangle$ with a reverse STIRAP pulse. The number of recovered Feshbach molecules is counted. However, we only detect atoms that end up in the lowest Bloch band after dissociation of the Feshbach molecules (see Appendix). The oscillation can be understood as follows. We consider the localized spatial centerof-mass (c.o.m.) wavepacket of a Feshbach molecule at a particular lattice site in the lowest Bloch band. The first STIRAP transfer projects this wavepacket onto the much shallower $3^{3}$ lattice potential felt by the $|g\rangle$ molecules (Fig. (5) without changing its shape. As a consequence, $|g\rangle$ molecules are coherently spread over various Bloch bands, and the wavepacket undergoes "breathing" oscillations with the lattice site trap frequency $\omega_{t}$.

\footnotetext{
${ }^{3}$ Due to a smaller dynamic polarizability, the lattice depth for the tightly bound $|g\rangle$ molecules is shallower than for the Feshbach molecules by a factor of $\approx 10$.
} 
These coherent oscillations (period $\approx 80 \mu \mathrm{s}$ ) are damped by tunneling of $|g\rangle$ molecules in higher Bloch bands to neighboring lattice sites. The reverse STIRAP transfer maps this periodic oscillation back to the Feshbach molecule signal in Fig. 4. Higher Bloch bands are populated here as well, but are at most partially counted in our scheme (see Appendix), which leads to an apparent decrease in transfer efficiency.

The question arises why similar oscillations are not observed in our square pulse projection measurements shown in Fig. 2, especially for the case $\Omega_{1}=\Omega_{2}$ where $50 \%$ of the population is in state $|g\rangle$. One might assume that the spatial wavepackets of the $|g\rangle$ molecules undergo similar breathing oscillations. These oscillations would then periodically break up the dark superposition state and lead to corresponding losses. They would also periodically produce population in higher Bloch bands of the Feshbach molecule lattice. As we will see, the oscillations are suppressed because the Raman lasers phase lock the involved quantum levels which stops, in a sense, the free evolution of the wavepackets. We can understand this behavior in detail with the help of a multi-band model, which we describe in the following.

\section{Multi-band model}

In an optical lattice the molecular levels $|f\rangle,|g\rangle$ and $|e\rangle$ from the previous model have a substructure given by the lattice Bloch bands. Because the lattice depths for the levels $|f\rangle,|g\rangle$ and $|e\rangle$ are in general different, the respective band structures will also vary. This combination of external (c.o.m. motion in the lattice) and internal degrees of freedom gives rise to a number of new quantum levels which are coupled by the laser fields (Fig. 6). We assume each Feshbach molecule to be initially localized in a singly-occupied lattice site. The corresponding localized molecular wavepacket can be described by Wannier functions [15] which form a complete set of orthonormal functions. In the following we 


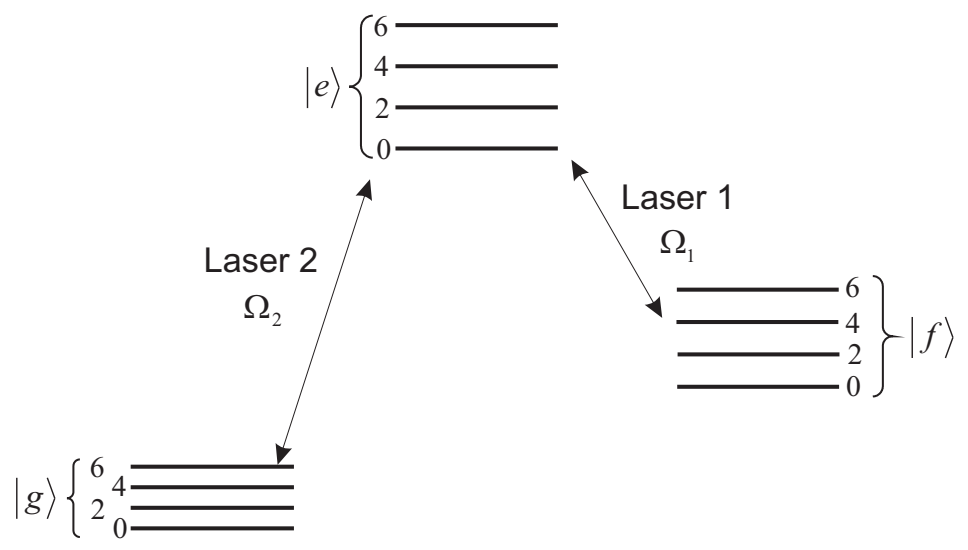

Figure 6: Multi-band model. The three molecular levels $|f\rangle,|e\rangle$ and $|g\rangle$ have a Bloch band substructure due to the optical lattice. We restrict the model to the 4 lowest Bloch bands with even symmetry (band index $n=0,2,4,6$ ).

will denote the Wannier function for level $|\alpha\rangle$ and band $n$ as $\left|\Psi_{\alpha n}\right\rangle$. We note that for deep lattices, these Wannier functions closely resemble harmonic oscillator wavefunctions.

The Raman lasers couple different $\left|\Psi_{\alpha n}\right\rangle$ according to the respective wavefunction overlaps (Fig. 6). Since the initial wavepackets of the Feshbach molecules are symmetric, only even bands will be populated. We restrict our calculations to the four lowest Bloch bands with even symmetry 4 corresponding to the band indices $n=0,2,4,6$. The dynamics in each of the three lattice directions is then described by a 12-level model, which can in principle be solved in terms of a master equation (Sec. 4). However, we have used a Schrödinger equation-based model since the numerical code is less involved. In this approach, laser phase fluctuations are neglected, and we introduce a lattice site tunnel rate for each band. These tunnel rates are chosen to match the expected tunnel rates for the different bands and are slightly adjusted for a better fit of the data in Fig. 4. We note that the results of the model calculations are essentially independent of the excited state lattice depth, which is not well known.

\footnotetext{
${ }^{4}$ The effect of including higher bands into the model was found to be negligible.
} 
The Hamiltonian $H$ of our time dependent Schrödinger equation

$$
i \hbar \frac{\partial}{\partial t}|\Phi\rangle=H|\Phi\rangle
$$

has the form of a $12 \times 12$ matrix,

$$
H=\hbar\left(\begin{array}{ccccc}
E_{f 0}-\frac{i}{2} J_{f 0} & 0 & \frac{1}{2} \Omega_{1}(t) \cdot M_{f 0, e 0} & 0 & \ldots \\
0 & E_{g 0}+\delta-\frac{i}{2} J_{g 0} & \frac{1}{2} \Omega_{2}(t) \cdot M_{g 0, e 0} & 0 & \ldots \\
\frac{1}{2} \Omega_{1}(t) \cdot M_{e 0, f 0} & \frac{1}{2} \Omega_{2}(t) \cdot M_{e 0, g 0} & E_{e 0}-\frac{i}{2} \Gamma-\frac{i}{2} J_{e 0} & \frac{1}{2} \Omega_{1}(t) \cdot M_{e 0, f 2} & \ldots \\
0 & 0 & \frac{1}{2} \Omega_{1}(t) \cdot M_{f 2, e 0} & E_{f 2}-\frac{i}{2} J_{f 2} & \ldots \\
\vdots & \vdots & \vdots & \vdots &
\end{array}\right)
$$

Here $E_{\alpha n}$ and $J_{\alpha n}$ are the energy and tunnel matrix element respectively for the Wannier function $\left|\Psi_{\alpha n}\right\rangle$ in band $n$ of level $|\alpha\rangle . M_{\alpha n, \beta k}=\left\langle\Psi_{\alpha n} \mid \Psi_{\beta k}\right\rangle$ is the overlap integral of the respective Wannier functions.

Diagonalizing this Hamiltonian, we find twelve "eigenstates" of the coupled system which in general have complex eigenvalues. In the following, we study the case of strong coupling $\left(\Omega_{1,2} \gg \omega_{t}\right), 5$ which is the regime for phase locking. In this regime, four of these eigenstates have negligible contribution from the exited level $|e\rangle$ and thus a long lifetime. These 4 quasi-dark states essentially correspond to the 4 lattice bands in our model and will be denoted as $\left|D S_{n}\right\rangle$ with $\mathrm{n}=0,2,4,6$. We now study the spatial waveforms of these dark states (Fig. (7) and compare the components with $|f\rangle$ and $|g\rangle$ character. Neglecting a small $|e\rangle$ component the dark superposition state, $\left|D S_{n}\right\rangle$ can be written as

$$
\left|D S_{n}\right\rangle=|g\rangle\left\langle g \mid D S_{n}\right\rangle+|f\rangle\left\langle f \mid D S_{n}\right\rangle .
$$

As an example $\left(\Omega_{1}=\Omega_{2}\right)$ Fig. 7 shows that the wavepackets $\left\langle g \mid D S_{n}\right\rangle$ and $\left\langle f \mid D S_{n}\right\rangle$ have the same shape. This is not surprising since this ensures that the ratio of the $|f\rangle$ and $|g\rangle$ amplitudes equals $\Omega_{2} / \Omega_{1}$ everywhere, as in Eq. 1.

\footnotetext{
${ }^{5}$ For our experiments where $\Omega_{1,2} \gtrsim 2 \pi \times 1 \mathrm{MHz}$ and $\omega_{t} \sim 2 \pi \times 10 \mathrm{kHz}$ this condition is satisfied.
} 


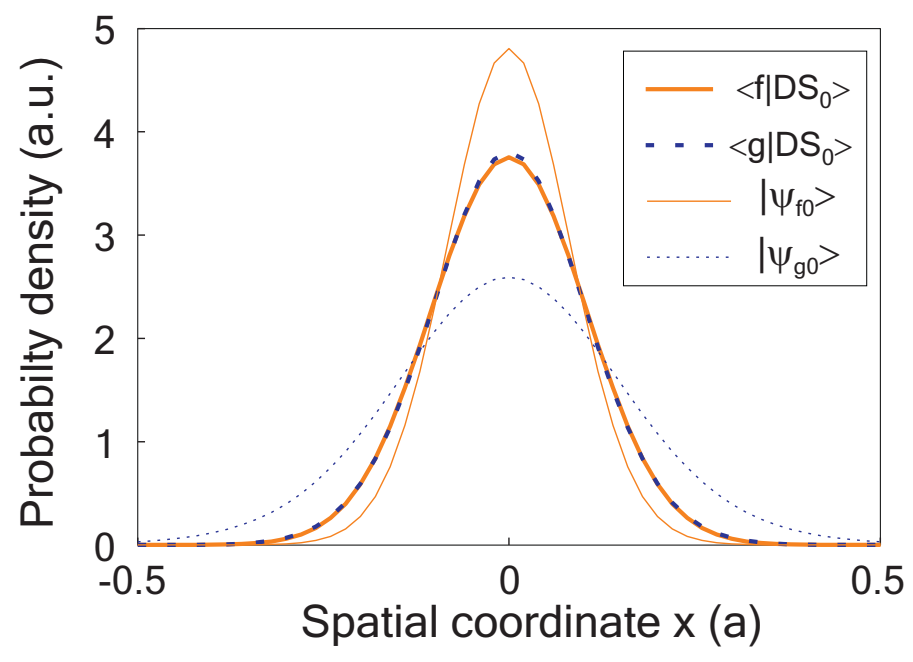

Figure 7: Spatial wavepackets of the dark state $\left|D S_{0}\right\rangle$ and the Wannier functions $\left|\Psi_{f 0}\right\rangle$, $\left|\Psi_{g 0}\right\rangle$. The dark state $\left|D S_{0}\right\rangle$ has two components, one having $|f\rangle$ character $\left(\left\langle f \mid D S_{n}\right\rangle\right)$ and the other one having $|g\rangle$ character $\left(\left\langle g \mid D S_{n}\right\rangle\right)$. The wavepackets of $\left\langle f \mid D S_{n}\right\rangle$ and $\left\langle g \mid D S_{n}\right\rangle$ essentially have the same shape. They are mainly composed of the lattice ground states $\left|\Psi_{f 0}\right\rangle$ (thin solid line) and $\left|\Psi_{g 0}\right\rangle$ (thin dotted line). All depicted states are normalized. The parameters used are $\Omega_{1}=\Omega_{2}=2 \pi \times 7 \mathrm{MHz}, V_{f}=60 E_{r}$ and $V_{g}=6 E_{r}$, as in our experiments.

Let us now discuss the formation and evolution of the dark state that we have observed in the square pulse experiments of Sec. 3. A dark state $|D S\rangle$ is formed in less than $1 \mu$ s by subjecting Feshbach molecules to a square Raman laser pulse. As in the STIRAP transfer (discussed in Sec. 5) the initial projection onto $|D S\rangle$ will not change the shape of the Feshbach molecule wavepacket, given by the Wannier function $\left|\Psi_{f 0}\right\rangle$. The dark state can be expressed as a coherent superposition of the four dark eigenstates $\left|D S_{n}\right\rangle$ of the 12-level Hamiltonian

$$
|D S\rangle=\sum_{n=0,2,4,6} c_{n}\left|D S_{n}\right\rangle
$$

The subsequent coherent evolution of these dark states will again in principle lead to breathing oscillations. The amplitude of these oscillations depends on the extent to which higher bands (i.e., $|D S\rangle_{n}, n>0$ ) are excited. The excitation increases with increasing deviation of $|D S\rangle$ from the initial state $|f\rangle$, i. e., with rising $\Omega_{1} / \Omega_{2}$. 
This can also be understood from another point of view. The effective lattice potential felt by the molecules in such a superposition state is the weighted average of the potentials for the two contributing states $|f\rangle$ and $|g\rangle$. For the case $\Omega_{1}=\Omega_{2}$ this effective potential is about half as deep as that for the Feshbach molecules. Compared to the case of pure ground state molecules (Fig. (4) where the lattice potential is reduced by a factor of 10 , the oscillations of the wavepacket are strongly suppressed and cannot be observed with our current experimental precision. For $\Omega_{1} \gg \Omega_{2}$, the dark state $|D S\rangle$ has a dominant contribution from state $|g\rangle$, and the effective lattice potential essentially corresponds to the one for ground state molecules. In this case oscillations appear despite the strong coupling, a fact which we also have experimentally verified 6 .

Conclusion We have analyzed coherent wavepacket dynamics and their suppression in a 3D optical lattice. We observed optically induced phase locking of a number of quantum levels, which can also be viewed as the appearance of a novel multi-level dark state. The experiments were carried out with tightly bound molecules as a component of a dark quantum superposition state. Thus, the experiments demonstrate control of molecular motion in an optical lattice for the first time. In addition, different models have been introduced and discussed in detail, with which the lattice dynamics can be understood and quantitatively described.

Acknowledgements The authors thank Helmut Ritsch for very helpful discussions and support in model calculations. We also thank Gregor Thalhammer for early assistance in the lab, and Florian Schreck for loaning us a Verdi V18 pump laser. This work was supported by the Austrian Science Fund (FWF) within SFB 15 (project part 17).

\footnotetext{
${ }^{6}$ For these experiments we have ramped into the dark state and back in a fashion similar to STIRAP transfer pulses to avoid strong losses caused by direct projection into $|D S\rangle \approx|g\rangle\langle g \mid D S\rangle$.
} 


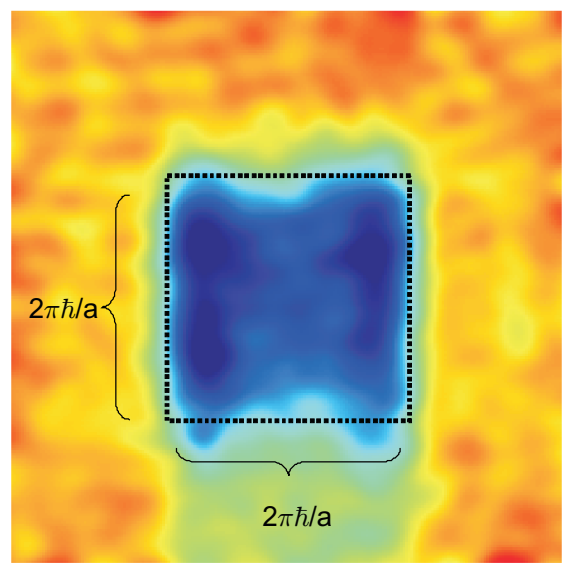

Figure 8: Shown is a typical absorption image which displays the atomic quasimomentum distribution in the optical lattice after exposure to the Raman laser beams and subsequent adiabatic molecule dissociation. Atoms inside the square region come from the lowest Bloch band. $2 \pi \hbar / a$ is the modulus of the reciprocal lattice vector.

\section{Appendix: Theoretical band population analysis}

As stated before, our signals only include molecules for which the constituent atoms end up in the lowest Bloch band of the lattice. A controlled lattice rampdown in a few milliseconds maps the bands and quasi-momentum distribution of the atoms into momentum space [16, 12]. We image these distributions after $13 \mathrm{~ms}$ of time-of-flight via absorption imaging. Fig. 8 shows a typical distribution. The dotted square region corresponds to the lowest Bloch band and is dominantly populated.

An important question is how the Bloch bands for the Feshbach molecules map onto the Bloch bands for the atoms. In other words, if we measure the atomic population of the Bloch bands - do we know what the band population for the molecules was? As the lattice is very deep for the Feshbach molecules and atoms, we can approximate the potential at an individual lattice site as harmonic with trap frequency $\omega_{t}$. In one dimension, the 
eigenfunctions of the harmonic oscillator are

$$
\left|\Phi_{n}\right\rangle=\frac{1}{\sqrt{2^{n} n ! \sqrt{\pi} x_{0}}} \exp \left(-\frac{1}{2}\left(\frac{x}{x_{0}}\right)^{2}\right) \mathrm{H}_{n}\left(\frac{x}{x_{0}}\right)
$$

where $x_{0}=\sqrt{\hbar / \omega_{t} m}$ is the oscillator length and $\mathrm{H}_{n}$ is the $\mathrm{n}^{\text {th }}$ Hermite polynomial. We assume that we have two atoms in a lattice site with coordinates $x_{1,2}$. The relative and c.o.m. coordinates of the atom pair are

$$
\begin{aligned}
& x_{r}=1 / \sqrt{2}\left(x_{1}-x_{2}\right) \\
& x_{c}=1 / \sqrt{2}\left(x_{1}+x_{2}\right)
\end{aligned}
$$

The c.o.m. $\omega_{t}$. In one dimension, the eigenfunctions of the harmonic oscillator are We assume that we have two atoms in a lattice site with coordinates $x_{1,2}$. The relative and c.o.m. coordinates of the atom pair are

$$
\begin{aligned}
& x_{r}=1 / \sqrt{2}\left(x_{1}-x_{2}\right) \\
& x_{c}=1 / \sqrt{2}\left(x_{1}+x_{2}\right)
\end{aligned}
$$

The c.o.m. potential $V_{c}$ for the pair will be harmonic with trap frequency $\omega_{t}$ and the potential $V_{r}$ for the relative coordinate will be a sum of the harmonic potential and the interaction potential (Fig. 9).

When we form or dissociate a molecule by adiabatically ramping across a Feshbach resonance, only the quantum level in the $V_{r}$ potential will change - from a molecular bound state to an unbound atomic pair state in the lowest Bloch band. The wavefunction in the c.o.m. coordinate remains unchanged. We can now calculate how band populations of Feshbach molecules converted to atomic band populations by using the coordinate transformations. As an example: A Feshbach molecule in the lowest Bloch band (i. e., center-of-mass coordinate) will produce an atom pair with the following wavefunction: 

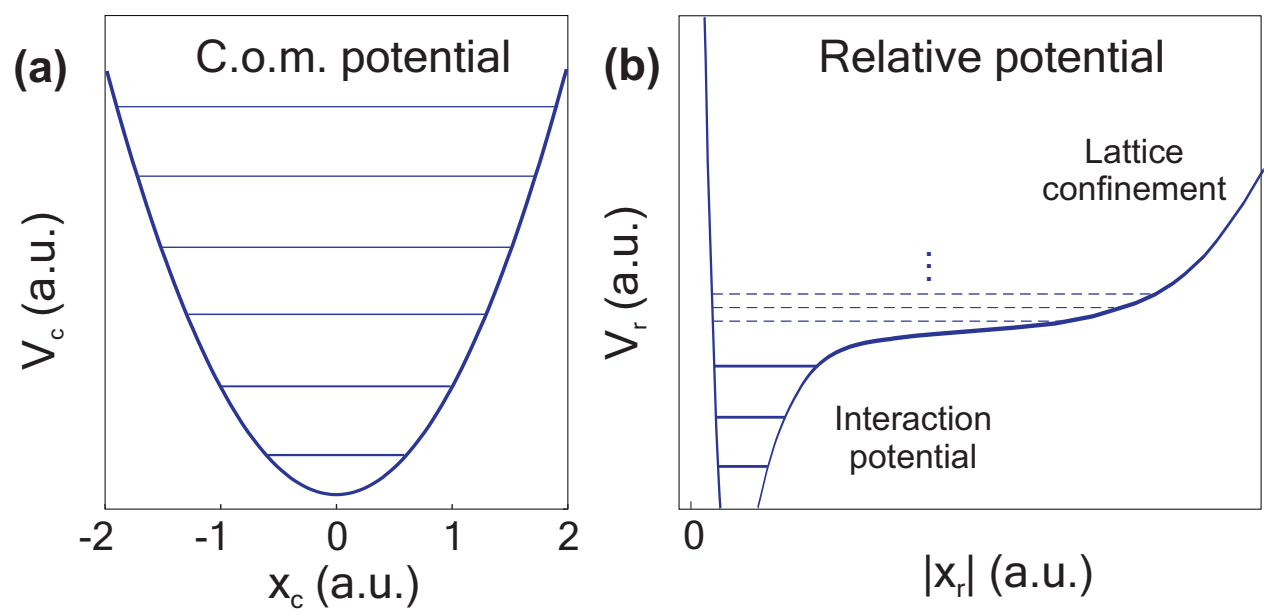

Figure 9: Potentials for the center-of-mass and relative coordinate of two atoms trapped at a site of the optical lattice. (a) The bound states (solid lines) of the harmonic centerof-mass potential $V_{c}$ correspond to the molecular Bloch bands. (b) At short interatomic distances the relative potential $V_{r}$ is dominated by the interaction potential, which allows the formation of bound molecular states (solid lines). Unbound atoms are trapped by the lattice potential at larger separation (dashed lines). With the help of a Feshbach resonance the lowest trap state can be converted into a high molecular state. Note that in this schematic view both energy and distance for the two contributions to the relative potential are not to scale.

$|\Psi\rangle \propto \exp \left(-1 / 2 x_{c}^{2}\right) \exp \left(-1 / 2 x_{r}^{2}\right)=\exp \left(-1 / 2 x_{1}^{2}\right) \exp \left(-1 / 2 x_{2}^{2}\right)$. This means that both atoms will also end up in the lowest Bloch band of the lattice. This analysis can be extended to any band. Table 1 gives the conversion amplitudes from molecular to atomic bands for the four lowest symmetric molecular bands. Correlations between the two constituent atoms of a molecule are not discussed here.

We finally note that when we apply absorption imaging, the optical density of the atomic sample is integrated in the direction of observation. Thus in this direction no band population analysis is possible. We accounted for this in our multi-band model described in Sec.6, 


\begin{tabular}{|c|c|c|c|c|c|c|c|c|c|c|}
\hline & \multicolumn{9}{|c|}{ atomic Bloch band } \\
\hline & & 0 & 2 & 4 & 6 & 8 & 10 & 12 & 14 & $\ldots$ \\
\hline \multirow{5}{*}{ 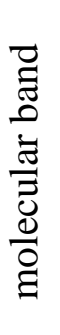 } & 0 & 1 & 0 & 0 & 0 & 0 & 0 & 0 & 0 & \\
\hline & 2 & $\frac{1}{2}$ & $\sqrt{\frac{1}{2}}$ & $\frac{1}{2}$ & 0 & 0 & 0 & 0 & 0 & .. \\
\hline & 4 & $\frac{1}{4}$ & $\sqrt{\frac{3}{16}}$ & $\sqrt{\frac{1}{2}}$ & $\sqrt{\frac{3}{16}}$ & $\frac{1}{4}$ & 0 & 0 & 0 & \\
\hline & 6 & $\frac{1}{8}$ & $\sqrt{\frac{3}{64}}$ & $\sqrt{\frac{3}{16}}$ & $\sqrt{\frac{1}{2}}$ & $\sqrt{\frac{3}{16}}$ & $\sqrt{\frac{3}{64}}$ & $\frac{1}{8}$ & 0 & $\cdots$ \\
\hline & $\vdots$ & $\vdots$ & $\vdots$ & $\vdots$ & $\vdots$ & $\vdots$ & $\vdots$ & $\vdots$ & $\vdots$ & \\
\hline
\end{tabular}

Table 1: Band conversion amplitudes in the harmonic oscillator approximation. Each line gives the amplitudes for a constituent atom of a molecule in a certain band to populate various atomic bands after dissociation. 


\section{References}

[1] F. Lang, K. Winkler, C. Strauss, R. Grimm, J. Hecker Denschlag Phys. Rev. Lett., 2008, 101, 133005.

[2] M. Viteau et al., Science, 2008, 321, 232.

[3] J. G. Danzl et al., Science, 2008, 321, 1062.

[4] K.-K. Ni et al., Science, 2008, 322, 5899.

[5] J. Deiglmayr et al., Phys. Rev. Lett., 2008, 101, 133004.

[6] K. M. Jones, E. Tiesinga, P. D. Lett, P. S. Julienne, Rev. Mod. Phys., 2006, 78, 483.

[7] J. M. Hutson, P. Soldán, Int. Rev. Phys. Chem., 2006, 25, 497.

[8] T. Köhler, K. Goral, P. S. Julienne, Rev. Mod. Phys., 2006, 78, 1311.

[9] K. Bergmann, H. Theuer, B. W. Shore, Rev. Mod. Phys., 1998, 70, 1003.

[10] G. Thalhammer et al., Phys. Rev. Lett., 2006, 96, 050402.

[11] T. Volz, S. Dürr, S. Ernst, A. Marte, and G. Rempe, Phys. Rev. A, 2003, 68, 010702(R).

[12] K. Winkler et al., Nature, 2006, 441, 853.

[13] D. F. Walls, G. J. Milburn, in Quantum Optics, Springer-Verlag, Berlin, 1994.

[14] T. Haslwanter, H. Ritsch, J. Cooper, and P. Zoller, Phys. Rev. A, 1998, 38, 5652.

[15] W. Kohn, Phys. Rev. B, 1973, 7, 4388.

[16] J. Hecker Denschlag et al., J. Phys. B: At. Mol. Opt. Phys., 2002, 35, 3095. 